\title{
Subcutaneous nodules, a rare presentation of sarcoidosis
}

\author{
Sumeet David $^{1 *}$, GR Garg ${ }^{2}$, Nitin Malhotra ${ }^{3}$ \\ ${ }^{1}$ Assistant Professor Department of Medicine, CMC \& H, Ludhiana \\ ${ }^{2}$ Assistant Professor, Department of Medicine, CMC \& H, Ludhiana \\ ${ }^{3}$ Professor, Department of Medicine, CMC \& H, Ludhiana \\ *Corresponding author E-mail: sumeetdavid_2007@yahoo.co.in
}

Copyright (c) 2014 Dr. Sumeet David et al. This is an open access article distributed under the Creative Commons Attribution License, which permits unrestricted use, distribution, and reproduction in any medium, provided the original work is properly cited.

\begin{abstract}
A 53 year old lady was apparently well about 2 months back when she started complaining of painless subcutaneous swellings over the face and both the upper limbs.No systemic involvement was present .Histopathology of the lesion showed sarcoidosis.Patient responded to steroids .Serum ACE level was also raised(95). Subcutaneous nodules a rare presentation of sarcoidosis is discussed.
\end{abstract}

Keywords: Subcutaneous Nodules, Joint Pains, ACE Levels, No Systemic Involvement, Steroids Responsive.

\section{Case Report}

53 year old lady was apparently well about 2 months back when she started complaining of painless subcutaneous swellings over the face and both the upper limbs. She complained of stiffness of small joints of both hands in the morning for more than 1 hour duration.

In addition she had pain in both ankle and knee joints. There were multiple nodules ranging from 1-3 $\mathrm{cm}$ in both the upper limbs and lateral aspect of the left eyebrow. Firm, non-tender, non adherant to skin and subcutaneous tissues.No joint deformity was present. Rest of the systemic examination was

Normal. Hematological and biochemical investigations were normal except ACE levels are raised.Biopsy done showed non caseating granulomatous inflammation suggestive of sarcoidosis.

This figure shows picture of forearm showing showing subcutaneous nodule right forearm (figure a).

This figure shows histopathological features of subcutaneous sarcoidosis right forearm (figure b-b1,b2,b3).

\section{Discussion}

Subcutaneous sarcoidosis is a rare, specific subtype of nodular cutaneous sarcoidosis that was originally described by Darier and Roussy in 1904. Although these nodules are often referred to as Darier-Roussy sarcoid, this is actually a term that is used to describe many subcutaneous inflammatory disorders and is therefore non-specific [1]. Diagnosis is made by demonstration of non-infectious sarcoidal or non-caseating epithelioid granulomas with multinucleated giant cells with lymphocytic infiltration mainly involving the panniculus [1], [2] often associated with systemic involvement especially hilar lymphadenopathy. However subcutaneous nodules may be seen without systemic involvement. Subcutaneous sarcoidosis presents as painless, firm, mobile nodules without overlying epidermal involvement [3]. These nodules have a predilection for the trunk and extremities, but also occur on the face. The range of the number of lesions is 1 to 100 , and the average size of these lesions is 1 to $2 \mathrm{~cm}$ [4]. A large retrospective and prospective study found 54 cases [5]. Peak incidence of subcutaneous sarcoidosis in the fourth decade of life and is slightly higher in female patients as compared to male patients. Association of subcutaneous sarcoidosis with autoimmune disorders like vitiligo, pernicious anemia and thyroiditis has been described [5], [6]. Another, smaller retrospective review that included 10 patients with subcutaneous sarcoidosis showed preponderance of female patients in the fifth and sixth decade of life [7].This patient presented to us subcutaneous swelling which on histopathology showed sarcoidosis 
.These subcutaneous nodules responded to steroids.Other drugs which can be used for this condition are minocyline and doxycycline.

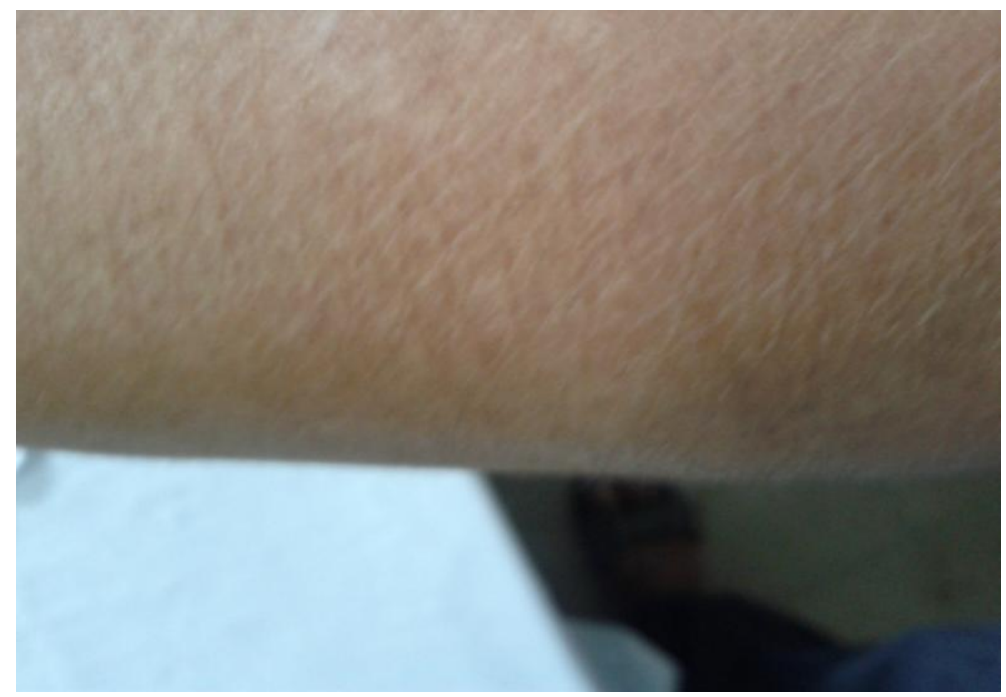

Fig. a: Subcutaneous nodules

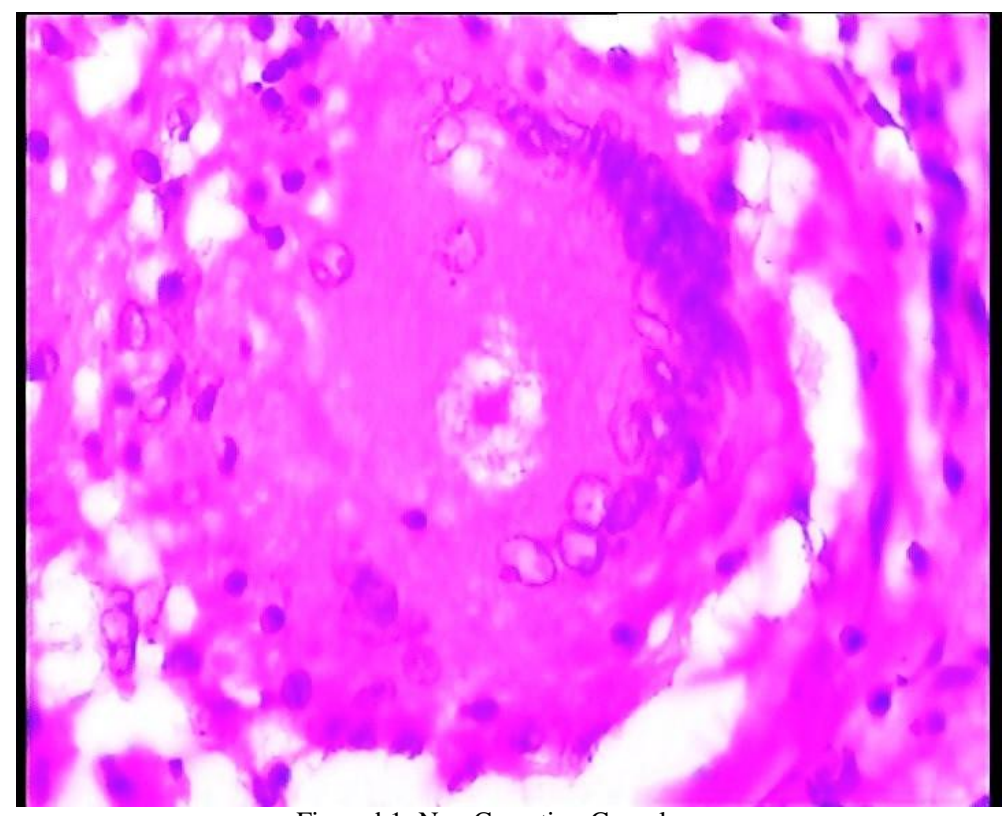

Figure b1: Non Caseating Granuloma.

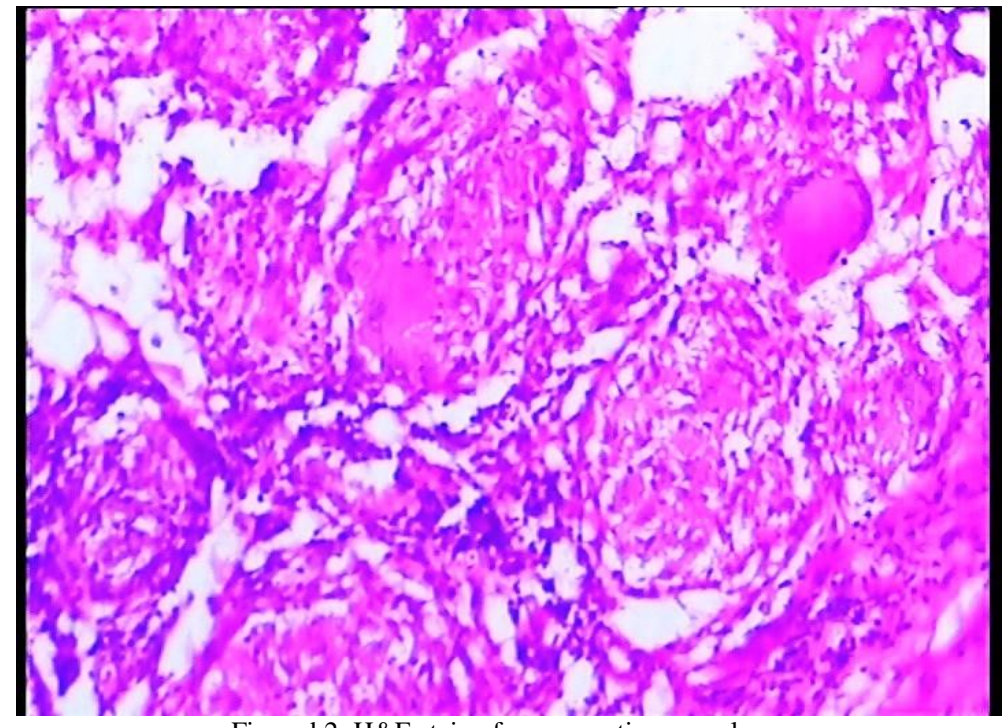

Figure b2: H\&E stain of non caseating granuloma 


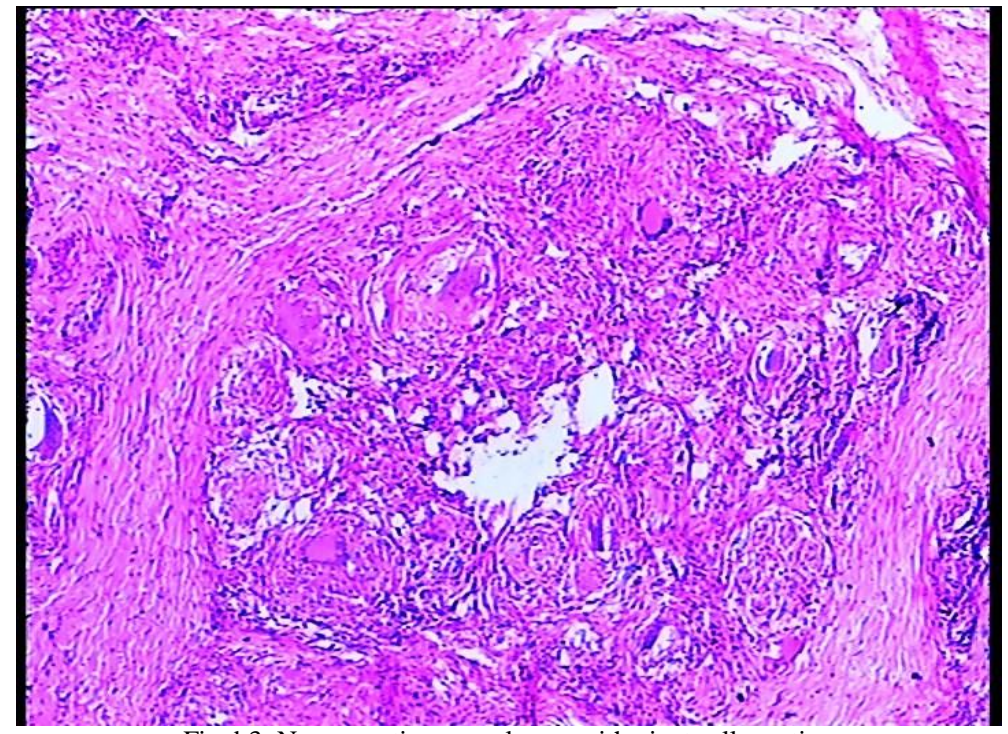

Fig. b3: Non caseating granulomas with giant cell reaction

$\mathrm{H} \& \mathrm{E}$ stain of the nodule showing closely packed, Non-caseating Hyalinising Granulomas with giant cell reaction consistent with sarcoidosis.

\section{Conclusion}

subcutaneous nodules presenting as sarcoidosis is one of the rare presentation there is no systemic involvement which can be confirmed by histopathological examination which responds to steroids. Other drugs which can be given minocycline and doxycycline.

\section{References}

[1] Vainsencher D, Winkelmann RK. Subcutaneos sarcoidosis. Arch Dermatol 1984; 120:1028.

[2] Shapiro PE. Noninfectious granulomas. In: Elder D, et al, eds. Lever's Histopathology of the Skin. 8th edition. Philadelphia: LippincottRaven, 1997:323.

[3] Howard A, White CR. Non-infectious granulomas. In:Bolognia JL, et al, eds. Dermatology. New York:Mosby, 2003:1455.

[4] Kalb RF, et al. Sarcoidosis with subcutaneous nodules. Am J Med 1988; 85:731.

[5] Ahmed I, Harshad S. Subcutaneous sarcoidosis: is it a specific subset of cutaneous sarcoidosis frequently associated with systemic disease? J Am Acad Dermatol 2006; 54:55.

[6] Barnadas MA, et al. Subcutaeous sarcoidosis associated with vitiligo, pernicious anemia and auto immune thyroidosis. Clin Exp Dermatol 2000: 25:55.

[7] Marcoval J, et al. Subcutnaeous sarcoidosis- clinicopathology study of 10 cases. Br J Dermatol 2005; 153-790. 